\title{
Foundation dentists' experiences and their contribution to vaccination programmes
}

\author{
Foundation dentists supporting vaccination programmes in England. Br Dent J 2021; https://doi.org/10.1038/s41415-021-3758-5
}

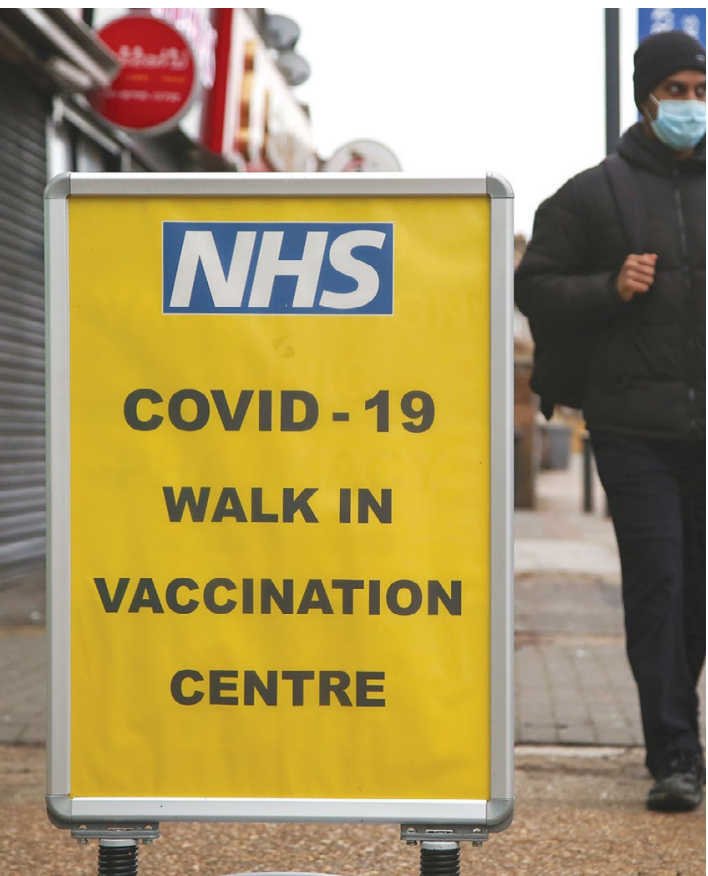

Dentistry provided under the National Health Service (NHS) has been greatly impacted by COVID-19. Additional measures to mitigate the risk of COVID-19 transmission, staff shortages and mounting workforce pressures led to reductions in patient attendances for dental treatment. This posed training challenges for newly qualified foundation dentists (FDs). To support the NHS, a working group consisting

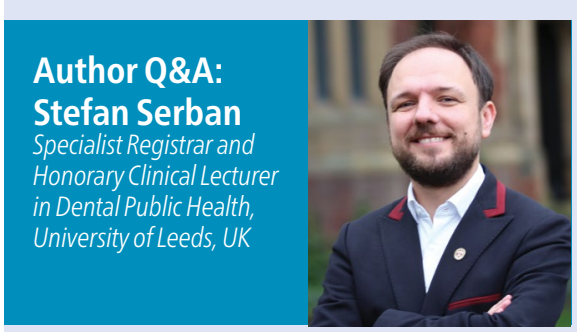

Why did you decide to undertake this study? In autumn 2020, the expansion of the eligible cohorts for the flu vaccination programme and later, the introduction of the COVID19 vaccination programme, highlighted a significant workforce capacity gap in our region. At the same time, because of the new restrictions caused by the pandemic, many foundation dentist (FD) training practices were struggling to offer more than three days/ of FDs from Yorkshire and the Humber was developed to aid COVID-19 and influenza vaccination programmes. FDs were trained and assessed before being invited to participate in vaccination programmes for one day per week over a six-month period (October 2020 to April 2021).

The experiences of redeployed FDs and stakeholders involved in the mobilisation of FDs were ascertained through surveys and interviews, resulting in a mixed-methods service evaluation.

In total, $106 \mathrm{FDs}$ were trained in delivering vaccinations. The questionnaire of the FDs had a response rate of $89 \%(n=94)$. Surprisingly, $40 \mathrm{FDs}$ were not utilised for vaccination, despite being trained. A number of FDs attributed this to a lack of clarity regarding the role of the FDs within the vaccination programmes and logistical reasons (for example, being asked to vaccinate at short notice).

Interviews were undertaken to establish the views of stakeholders from six organisations including Public Health England and Health Education England. Eleven participants were questioned on their views of the value of utilising FDs as well as possible barriers.

Stakeholders reported positively on the value of opportunities for FDs to work in a different environment and learn new skills. A few expressed concerns about the lack

week of work to the FDs. We had to do something to help, but these were uncharted waters and there was no precedent in terms of governance arrangements. Fortunately, our team was led by a dental public health consultant with a dual role as screening and immunisations lead. We also had excellent relationships already established with key partner organisations including Health Education England, NHS England, Y\&H Academic Health Sciences Network etc.

Did any of the results surprise you?

There's no surprise that dentists can vaccinate; the surprising part was around the ability of different organisations and systems to work together. Some were innovators and early adaptors who jumped on the free offer whilst others needed a little bit more support. of experience of FDs exacerbated by the pandemic. It was recognised that redeployment of FDs enabled primary care staff to focus on primary care work.

Eight participants reported motivating people to engage and resistance to change as potential barriers. Other reported factors included complex employer protocols, competency sign-offs, the absence of an existing framework, and a lack of understanding of the FDs' skill set and training to date.

A lack of understanding of the FDs' skill set was identified by both groups. This may be attributed to the detached delivery of dental training from other healthcare services. Overcoming this barrier may lead to better person-centred multidisciplinary care between healthcare professionals. Furthermore, this service evaluation has introduced the possibility of exploring dental care professional involvement in other aspects of healthcare and prevention (that is, vaccinations).

Although novel, the results of the evaluation would suggest that utilising FDs in vaccination programmes has been successful. Further efforts are required to combat identified barriers, including a lack of understanding about professional skill sets.

By Hasneet Kalsi

Specialist Registrar in Restorative Dentistry, Glasgow Dental Hospital, UK

Colleagues in the wider healthcare sector are not always aware of the skillset of dental teams. Fortunately, we had the support of some senior colleagues who understood the situation and helped us drive change.

What do you think the next steps should be considering your findings?

Dental access has to be the main priority for the moment. But in a post-pandemic world, there are lessons to be learned in working across traditional professional boundaries, especially with the increasing role of integrated care systems. We are looking to further explore opportunities around addressing the barriers between medical and dental teams as we cannot talk about health without considering oral health as an integral part of it. 